\title{
Una comunidad de seguridad regional en formación: la UNASUR y su Consejo de Defensa
}

\author{
A regional security community in the making: \\ UNASUR and its South American Defence Council
}

\author{
Daniel Flemes", Detlef Nolte ${ }^{* *}$ y Leslie Wehner ${ }^{* * *}$
}

\section{Resumen}

Este artículo analiza la formación y el proceso sostenido de institucionalización formal e informal de la UNASur y su Consejo de Defensa mediante el discurso y la acción, conceptualizados como una comunidad de seguridad regional. Se establece que ambos se encuentran al comienzo de un estado «ascendente» y que la intención de los países líderes del grupo que los forma es crear un ente regional de tipo «riguroso». Paradójicamente, las crisis son lo que crea un proceso discursivo que impone la necesidad de profundizar la UNASUR y la convierten en un mecanismo de equilibrio institucional y de poder frente a grupos regionales como la oEA y países como los Estados Unidos.

Palabras clave: comunidades de seguridad; integración regional; seguridad; unASur, Consejo de Defensa

\footnotetext{
" Investigador GIGA- German Institute of Global and Area Studies.<flemes@giga-hamburg.de>

** Vice president de GIGA- German Institute of Latin American Studies. <nolte@giga-hamburg.de>

**** Investigador GIGA- German Institute of Global and Area Studies. <wehner@giga-hamburg.de>
} 


\section{Abstract}

This article examines the continuing process of establishment and formal and informal institutionalization of UNASUR's and its Security Defence Council through action and discourse. We claim that both are in an «ascent» phase, and that the aims of the group's leading countries are to create a «rigorous» regional grouping. Paradoxically, evidence points to crises as main elements that drive these countries to strengthen the institution.Moreover, the role placed by UNASUR during crises becomes a mechanism for achieving institucional balance and balance pf power vis-à-vis the oAs and the United States..

KEYWORDS: security communities; regional integration, security, UNASUR, South American Defence Council

Recibido el 23 de noviembre de 2010; aceptado el 13 de octubre de 2011. 
Daniel Flemes, Detlef Nolte y Leslie Wehner • Una comunidad de seguridad regional...

\section{INTRODUCCIÓN}

La creación de la Comunidad de Naciones Sudamericanas consagrada en la declaración de Cusco en 2004 -rebautizada en 2007 como Unión de Naciones Sudmericanas (UNASUR), pone de relieve un tema importante en las relaciones interestatales de Sudamérica como los es la cooperación regional en materias de seguridad. Los propósitos centrales de la UNASUR son configurar un área de democracia, paz, cooperación, integración, identidad sudamericana, y desarrollo socioeconómico para sus Estados miembros. En cuanto a la seguridad, la institución persigue:

- la coordinación entre los organismos especializados de los Estados Miembros, teniendo en cuenta las normas internacionales, para fortalecer la lucha contra el terrorismo, la corrupción, el problema mundial de las drogas, la trata de personas, el tráfico de armas pequeñas y ligeras, el crimen organizado transnacional y otras amenazas, así como para el desarme, la no proliferación de armas nucleares y de destrucción masiva y el desminado;

- el intercambio de información y de experiencias en materia de defensa (ver Tratado Constitutivo de UNASUR, 2008).

Sin embargo, un grupo regional como UNASUR, que promueva y coordine el manejo eficiente de los temas de seguridad y defensa a través de su Consejo de Defensa Sudamericano
(CDs) dista mucho de ser internalizado por sus países miembros, pese a que tanto le primera como el segundo han construido un rol de mediador ante conflictos internos e interestatales como el cumplido en Bolivia en 2008, Ecuador en 2010, y Colombia-Venezuela en 2010.

$\mathrm{Al}$ analizar los objetivos para desarrollar una agenda de seguridad común bajo el alero de UNASUR, las distintas experiencias de cooperación y de resolución de conflictos, y los intereses nacionales de los países que tienen mayor peso estructural en esta región, cabe preguntarse en primer lugar si Sudamérica está avanzando hacia una agenda común de seguridad, segundo, qué factores impiden o facilitan la emergencia de una agenda de seguridad común y por último, qué se pretende alcanzar en relación con agentes e instituciones externas que pudiesen cumplir objetivos similares.

El argumento central de este artículo es que Sudamérica está aún lejos de constituir una comunidad de seguridad madura en el sentido propuesto por Karl Deutsch (1957) y re-elaborado por Emmanuel Adler y Michael Barnett (1998). Siguiendo la tipología de comunidades de seguridad puede decirse que los países de la UNASUR pretenden establecer una comunidad de seguridad rigurosa, pero aún se encuentra en la etapa ascendente de su evolución. Se sostiene además que pese a los esfuerzos por generar una identidad común en materia de seguridad -mediante la adopción de prácticas y hábitos de se- 
guridad, tales como el uso de la UNASUR como plataforma para la resolución de conflictos y el uso discursivo unificador de Estados Unidos como un ente que debe ser excluido de la región para legitimar la existencia y desarrollo de la UNASUR y su CDS- existen intereses materiales e ideológicos divergentes que impiden una mayor profundización de la cooperación.

Existen agendas nacionales individuales, la más destacada de las cuales es la de Brasil, que persigue ejercer un rol de poder a nivel global, pero también hay factores de división de carácter ideológico, como los propuestos por Venezuela-Bolivia (socialismo del siglo XXI) frente a países que siguen un modelo aperturista en lo político-económico y mantienen mayores vínculos con actores externos, como Colombia, Perú y Chile. Así, hay modelos autonomistas y otros que, sin renunciar a una mayor autonomía decisoria frente a países como los Estados Unidos, en el área de la defensa buscan mantener vínculos institucionales con actores externos a la región.

Es importante estudiar la UNASUR y su CDS como órgano de cooperación en temas de defensa y seguridad no solo por la novedad de su enfoque e intencionalidad, que expresan una creciente madurez así como la necesidad de sus miembros de coordinarse ante desafíos de interés común, sino también por la competencia, superposición o eventual complementariedad funcional que producen o podrían producir launASUR y el CDs respecto de la Organización de los
Estados Americanos (OEA) en materia de membresía y prácticas de seguridad.

Sin embargo, el cDs todavía es un foro de discusión y de proyección de experiencias bilaterales sobre defensa y seguridad más que un ente articulador de políticas regionales concretas en estos ámbitos. Sin embargo, la participación de la UNASUR en la resolución de conflictos regionales ha permitido articular un discurso que hace necesaria una mayor institucionalización del grupo como organización cuyo rol es mantener la paz y la democracia en la región. Así pues, la UNASUR está en un proceso sostenido de evolución hacia la búsqueda de roles, identidad e institucionalización.

A continuación se da una mirada general a la UNASUR y el CDS y se exponen algunos de sus desafíos actuales. Luego se presenta un marco analítico que entrelaza de manera ecléctica elementos de las comunidades de seguridad del neorrealismo y el institucionalismo, así como del constructivismo; en especial las ideas de poder (intereses nacionales) y de balance institucional, y del rol del discurso como ente articulador de la necesidad de instituciones de seguridad regionales. En una cuarta sección se analizan primero las agendas nacionales particulares de países como Brasil, Venezuela, Argentina, Chile y Colombia, en el caso del primero en su condición de poder regional y de poderes regionales intermedios de los demás, para luego examinar algunos episodios de crisis en que ha participado la UNASUR. Además, se analiza cómo 
Daniel Flemes, Detlef Nolte y Leslie Wehner • Una comunidad de seguridad regional...

estas crisis generan un patrón de balance institucional y de desplazamiento a partir de instituciones como la oEA y de actores externos como Estados Unidos. También se examina cómo este rol de manejo de crisis crea discursivamente la necesidad de esta institución como ente encargado de administrar y coordinar las agendas de seguridad en la región.

\section{Desafíos actuales de la UNASUR Y SU CDS}

Dentro de la UnASur, el ente rector de los temas de seguridad y defensa es el CDS. La finalidad de este Consejo es generar mecanismos de gobernanza para un vasto espectro de temas. El CDS es en sí una instancia de consulta, cooperación y coordinación en materias de defensa. De hecho, la unAsur en general y el CDs en particular no solo buscan crear un espacio institucional funcional para resolver conflictos sino también desarrollar una identidad común en el sentido de «nosotros los sudamericanos», que logre facilitar la cooperación y que por ende lleve a soluciones pacíficas y «regionales» de conflictos internos, interestatales o generados por grupos no- estatales ligados al tráfico de drogas, grupos paramilitares y subversivos (Statute of the Unasur South American Defense Council, 2008).

Entre los hitos fundamentales del CDS cabe señalar las declaraciones de la UNASUR en las reuniones extraordinarias de jefes de Estado y de Gobierno, como la de San Carlos de Bariloche en agosto de 2008, y de Ministros de Defensa y Relaciones Exteriores en Quito, en septiembre y noviembre de 2009, respectivamente. En la primera de ellas se proclamó a Sudamérica como Zona de Paz y los países de la UNASUR se comprometieron a establecer un mecanismo para desarrollar la confianza mutua en el ámbito de seguridad y defensa. En la segunda, el Canciller de Perú sugirió que se suscribiera un pacto de no agresión militar, que fue rechazado por Chile y otros países. Sin embargo, los mandatarios de los países miembros están estudiando un documento sobre medidas de confianza y seguridad que sigue los lineamientos de la reunión de Bariloche.

En la tercera reunión, realizada en noviembre de 2009 , se establecieron las medidas de desarrollo de confianza mutua y seguridad. Entre ellas se cuentan el intercambio de información y la transparencia de los sistemas de seguridad y de los gastos militares de cada país; la promoción de actividades militares tanto intra como extrarregionales; medidas de seguridad como el control y vigilancia de las fronteras y la prevención conjunta de la presencia y proliferación de grupos armados no reconocidos por la ley; medidas de garantía como la declaración de Sudamérica como zona libre de armas nucleares, así como el rechazo al uso de fuerza militar contra un Estado soberano y su integridad territorial; y medidas de cumplimiento y verificación de los compromisos mediante visitas a 
instalaciones militares y constatación de la situación en las distintas fronteras (ver Consejo de Defensa Suramericano, 2009a).

Si bien estas intenciones y objetivos de la UNASUR y del CDS son en sí loables muchas veces os intereses nacionales de sus miembros inhiben el proceso de su materialización plena. Por ejemplo, en Brasil, Venezuela y Chile el aumento de las compras de equipo militar genera suspicacias y desconfianzas bilaterales que se llevan al plano regional en UNASUR. En relación con el PIB, en 2007 el gasto en defensa de Brasil fue del 1,5\% -en comparación con un $4 \%$ en Estados Unidos, Destaca el caso de Chile que, con un $3,4 \%$ supera ampliamente a Argentina cuyos gastos en defensa representan solo un $0,8 \%$ del PIB. Los gastos de Colombia y Ecuador son bastante elevados ya que representan, respectivamente, el $11 \%$ y el $9 \%$ de los presupuestos de sus gobiernos. Además, casi todos los sistemas de defensa se importan de Rusia, Estados Unidos, Francia y Alemania (ver Flemes y Nolte, 2010: 5).

Las compras de armamento se traducen en desconfianzas bilaterales entre países vecinos, lo que puede convertirse en presión para que los demás países también incurran en gastos en esta área para mantener su poder disuasivo y los balances regionales. Además, con estas compras se generan alianzas tácitas como la de Venezuela y Rusia, y se acentúan las desconfianzas vecinales por la cooperación militar entre Colombia y Estados Unidos. Los acuerdos militares y de defensa con potencias extrarregionales terminan por sumarle una dimensión externa a la ya compleja agenda de seguridad regional. En este sentido, estas alianzas acentúan la polarización ideológica que, por ejemplo, ha llevado a fuertes desavenencias entre los países de la región por el tema de las bases militares estadounidenses en Colombia, lo que es visto con cierto escepticismo por Brasil, Argentina y Venezuela. Incluso países como Chile y Uruguay no ven con muy buenos ojos estas bases. La existencia de problemas con tintes ideológicos y de eventuales desequilibrios de poder, tiende a disminuir el compromiso por avanzar en una agenda de seguridad común en el CDS. Ejemplo de ello es la constante renuencia de Colombia entre 2008 y 2009 por integrarse al CDS y avanzar en una agenda de seguridad en común con sus pares situados al otro extremo ideológico -Venezuela y Ecuador (Flemes y Nolte, 2010: 5-7).

Por otra parte, existen otros temas de seguridad pendientes como el hecho de que Perú reclame por el creciente armamentismo en la región, apuntando principalmente hacia Chile, país con el que mantiene un litigio en la Corte de la Haya por la delimitación marítima (Flemes y Nolte, 2009). No hace mucho, Chile y Bolivia estaban envueltos en un juego retórico iniciado en Bolivia ante el inminente acuerdo de estos dos países para exportar gas boliviano por y para Chile. Esto llevó a la crisis del gas -bajo el mandato de Sánchez de Lozada en Bolivia (2002-03), que se convirtió 
Daniel Flemes, Detlef Nolte y Leslie Wehner • Una comunidad de seguridad regional...

en la llamada política de «gas por mar» durante la presidencia de Carlos Mesa (2003-05) (Wehner, 2010).

En cuanto a la dinámica interna de UNASUR y su CDSmás allá de su rol de mediación ante conflictos internos, que atentan contra la estabilidad democrática, e interestatales que amenazan la coexistencia pacífica, los resultados son ambiguos en el sentido de que en 2009 no avanzaron mayormente muchas de las actividades del plan de acción del CDS 2009-10, con excepción de la realización de un encuentro sobre Estudios estratégicos y de un seminario sobre Modernización de los Ministerios de Defensa (Ugarte 2010: 31).

Durante el año 2010, se llevaron a cabo una serie de seminarios y talleres de corte técnico y académico como parte del plan de acción 2010-11, entre los que cabe mencionar el seminario sobre Enfoques conceptuales en materia de defensa, riesgos y amenazas en la región y la participación de los ministerios de defensa y de las Fuerzas Armadas en caso de desastres naturales, y el taller sobre Industria y tecnología de la defensa. También se planificó llevar a cabo un ejercicio militar conjunto ante un escenario de catástrofes naturales en Perú durante la primera quincena de diciembre 2010, que finalmente se postergó (Presidencia Pro-Tempore de Ecuador 2010: 6, 16, Anexo 1).

También se creó un Centro de Estudios Estratégicos de Defensa del CDS cuya finalidad es crear una comunidad epistémica sudamericana en estos temas que trascienda el pensamiento nacional, pero que además sirva de órgano asesor del cDs cuando este así lo requiera. Sin embargo, su inauguración para fines de 2010 fue reprogramada para el año 2011. Por otra parte, el proceso de institucionalización formal de la UNASUR tuvo un lamentable tropiezo al fallecer repentinamente su Secretario General, Néstor Kirchner. Hasta el momento no existe claridad sobre el mecanismo definitivo para reemplazarlo.

No obstante las dificultades descritas en la ejecución de los planes de acción del CDS y los problemas entre los Estados de esta subregión, también existen agendas de cooperación bilateral en temas de defensa y seguridad que de hecho se utilizan en el marco del CDS como base para una mayor colaboración entre los países. A manera de ejemplo cabe citar el sistema de homologación de gastos de defensa diseñado por la Comisión Económica para América Latina y el Caribe (CEPAL), que ha sido puesto en práctica exitosamente por Chile y Argentina para trasparentar sus gastos militares (Flemes y Nolte, 2010).

Perú y Chile utilizarán un mecanismo similar de medición estandarizada de los gastos de defensa con el fin de dar mayor transparencia al intercambio de información. Este mecanismo incluye, además la eliminación de cualquier hipótesis de conflictote las respectivas doctrinas nacionales de defensa. Lo interesante de este acuerdo es que pese a que la raíz del problema es bilateral, Chile condicionó el proceso de homologación como parte esencial del 
marco multilateral que provee el CDS. De hecho, para este Consejo el acuerdo chileno-peruano de agosto de 2010 es un paso más en el proceso de institucionalización de sus labores como organismo regional y de su política de promoción de la confianza mutua. El CDS -declaración de Guayaquil en 2010- se comprometió, bajo el liderazgo de Argentina y Chile, a desarrollar una metodología para medir los gastos nacionales de defensa (Consejo de Defensa Suramericano, 2010a: 1).

También puede apreciarse un creciente esfuerzo de los miembros del CDS por coordinar sus agendas de seguridad. Casos concretos de ello son las reuniones periódicas de los ministros de defensa para readecuar el plan de acción para los años 2010-2011. Tanto Argentina como Chile organizaron dos talleres técnicos en 2010 para elaborar criterios técnicos y uniformes para una «Metodología común de medición de los gastos militares (Presidencia Pro-tempore de Ecuador 2010: 37 y Anexo 5). Dentro del plan de acción 2010-2011 y de la agenda de desarrollo de confianzas mutuas regionales, Argentina y Chile organizarán un ejercicio militar conjunto de los países de la UNASUR basado en un escenario de operaciones de paz (Consejo de Defensa Suramericano, 2010b). De hecho, Argentina y Chile ya tienen una fuerza binacional operativa para estos casos -la Fuerza de Paz Binacional Cruz del Surlo que hace suponer que muchas de las iniciativas desarrolladas o por desarrollar en UNASUR se basan en experiencias ya probadas a nivel bilateral, que luego son adecuadas al contexto regional. En este sentido, la UNASUR parece ser un nivel más, aunque preponderante, de un sistema de gobernanza permeable y flexible que posibilita la importación de procedimientos bilaterales en el área de la defensa y la seguridad (Flemes y Radseck, 2009).

\section{Comunidades de SEguridad Regional: Poder, INSTITUCIÓN Y Discurso}

En el mundo actual, los riesgos y amenazas en materia de defensa y seguridad son primeramente regionales, más que locales, nacionales o internacionales. En segundo lugar, como consecuencia de la globalización y de la creciente interdependencia las barreras estatales se han adaptado, dando mayor margen a intervenciones externas ante problemas de crisis institucionales, humanitarias y de la democracia. Esto no significa que la soberanía del Estado como pilar se haya disuelto en las relaciones internacionales sino que existe mayor predisposición a aceptar intervenciones conjuntas cumpliendo con normas sedimentadas en la estructura internacional como son los derechos humanos y la democracia. Por último -y relacionado con los dos primeros aspectos- las instituciones regionales están pasando a desempeñar un rol central en la asunción de los mandatos de seguridad que ostenta a grandes rasgos la institucionalidad de las $\mathrm{Na}$ ciones Unidas (Tavares 2010: 3-6). Esta 
Daniel Flemes, Detlef Nolte y Leslie Wehner • Una comunidad de seguridad regional...

institución muchas veces carece de los mecanismos y de la coordinación necesarios para actuar de manera rápida y eficiente ante un creciente número de problemas de seguridad. En este sentido, existe un espacio para construir entes regionales que generen no solo una fuente coordinadora de políticas de seguridad regional, sino que además una identidad de seguridad compartida por los distintos países participantes.

Desde el punto de vista analítico, al perseguir un bien regional común como lo es la seguridad para sus Estados miembros, los organismos o grupos regionales pueden concebirse como entes que evolucionan desde una organización funcional a una comunidad de seguridad con fuerte arraigo identitario. Los agentes que promueven la idea de una comunidad de seguridad la perciben como una forma de crear una zona de paz estable. Además, existen al menos dos tipos de comunidades de seguridad; la primera, es más bien flexible o laxa y la segunda, más rígida y rigurosa. Las primeras suponen un grado de institucionalización menor, ya que los Estados soberanos que la componen solo reducen sus expectativas a cambios pacíficos, mientras que las segundas tienen un grado mayor de institucionalización de prácticas y exhiben un principio de ayuda mutua, y su objetivo es generar una estructura sólida de gobernanza (Adler y Barnett, 1998).

Ambas formas de comunidades de seguridad evolucionan al menos en tres etapas:: «naciente», «ascendente» y «madura». En la primera de ellas los Estados comienzan a elaborar la forma de coordinar sus actividades para aumentar su seguridad mutua y así sentar las bases para un colaboración incremental a mediano plazo. En la segunda etapa aumenta la densidad de la colaboración y la y tiende a desaparecer la percepción de otros Estados como una amenaza a la seguridad nacional.. Es en esta fase donde se empieza a gestar una identidad colectiva de seguridad entre los Estados, ya que materializan las normas, prácticas y acciones coordinadas de la comunidad. La última etapa tiene lugar cuando las normas, prácticas y acciones de esa comunidad alcanzan un alto grado de complejidad, en que la base de la cooperación en seguridad es el principio de mutualidad. Es decir, la ayuda mutua y la constante consulta entre con fines de coordinación se tornan vuelven rutinarias y habituales para enfrentar los desafíos de seguridad (Adler y Barnett, 1998: 29-58; Bellamy, 2004: 8-9).

Pese a que las prácticas y acciones de los Estados y de la comunidad de seguridad -en cualquiera de sus formas- se co-constituyen (Adler y Barnett 1998), el agente juega un rol importante en la génesis y en dar forma a las prácticas y esferas de acción de una comunidad de seguridad. En este sentido sin agente no existe una comunidad de seguridad. Por ende, es esencial analizar los intereses que mueven a estos Estados a buscar mayor coordinación y cooperación en estas áreas, pero también los elementos que impiden una evolución dentro de una misma fase y la transición de una etapa de naciente a ascendente y de ascendente a madura. 
Los intereses de los Estados por generar un espacio o grupo regional en materias de seguridad pueden evaluarse en un sentido de poder material o ideacional-normativo. El primero se relaciona con los beneficios y costos materiales de liderar la formación de un ente que proporcione gobernanza regional a una serie de temas o problemáticas comunes. Generalmente, el que asume este rol articulador es el Estado, que es percibido como líder o que persigue desempeñar una función mayor dentro de una región. Sin embargo, para que tengan éxito este proceso de emergencia institucional y el posterior de desarrollo, el líder necesita de otros; es decir, de alianzas sólidas en qué sostener dicho proyecto (Nolte, 2009: 15). En este sentido existe una jerarquía de países que va desde poderes regionales o hegemónico-regionales a países intermedios que definen los límites y parámetros de la agenda integradora y el paso de dicha integración. Otro aspecto que dice relación con las agendas de poder, una vez que el grupo regional está funcionando, es el de las distintas agendas de cada Estado. Estos persiguen formar grupos regionales más para defender sus intereses nacionales que para llegar a formar un ente socializador de temas de defensa en un sentido de identidad regional. En este caso también pueden darse intereses comunes, pero la lógica de fondo son las alianzas para alcanzar un objetivo nacional más que regional.

Aplicando un patrón de carácter más ideacional-normativo, los países líderes buscan generar una serie de prácticas sociales dentro del grupo, así como importar sus normas e ideas a fin de que se utilicen en la institucionalización del grupo regional. Esto da cierta ventaja a los países que deciden las formas y fundamentos de la integración y la cooperación (Hurrell, 2005: 195; Nolte, 2009). Los grupos regionales emergen no solo por intereses materiales de Estados que buscan ejercer mayor influencia en un espacio regional delimitado sino que se acompaña de un discurso basado en generar la necesidad de esa institución. Acá es donde el elemento discursivo es clave no solo en la génesis del grupo sino también cuando el órgano ya ha sido creado, a fin de generar nuevas competencias y sostener la labor integradora y coordinadora del grupo regional en todos sus campos. Por lo tanto, el discurso en torno a la necesidad y las labores que lleva a cabo el grupo regional es netamente intencional y generado desde los grupos o líderes que hay mayor necesidad de que exista y se desarrolle una institución que tenga mayores competencias (sobre liderazgo y discurso: Nabers, 2010).

Cuando se habla de comunidades de seguridad en el sentido propuesto por Adler y Barnett (1998), la mirada analítica se inclina a estas comunidades en el sentido de búsqueda de institucionalidad y gobernanza de seguridad. Esta búsqueda de mejorar y sedimentar prácticas formales e informales de gobernanza en temas de defensa y seguridad es lo que sostiene a las comunidades de seguridad $y$, 
Daniel Flemes, Detlef Nolte y Leslie Wehner • Una comunidad de seguridad regional...

además, explica sus procesos de transición desde una forma naciente a una ascendente y de una ascendente a una madura. En consecuencia, cualquiera se la forma en que se encuentre la comunidad de seguridad, siempre tiene un objetivo y un diseño básico comunes, que es proporcionar gobernanza regional.

Puede hablarse de la UnASUR y del CDs en el sentido más clásico de la definición de institución internacional; es decir, como "gama de reglas conectadas y persistentes tanto formales como informales que buscan prescribir roles, restringir actividades y moldear las expectativas» (Keohane, 1989: $3)$. Sin embargo, por lo general estas instituciones se constituyen a la vez para integrar a países con una mirada similar y para restringir, excluir y delimitar el campo de acción de actores e instituciones que son percibidos como externos, pero que igualmente ejercen un rol en la región. Es decir, las agendas de seguridad también se utilizan para mantener las esferas de influencia y equilibrar institucionalmente a otros grupos en que uno o dos agentes ejercen un rol predominante (sobre balance institucional: $\mathrm{He}, 2008)$. Un ejemplo de este proceso de equilibrio institucional es la UNASUR y su cds, donde Brasil y Venezuela -por motivos distintos- buscan excluir a Estados Unidos, México y la oeA (Nolte, 2009: 19; Flemes y Wojczewski, 2010: 12).

$\mathrm{Al}$ equilibrar instituciones y agentes externos surge un proceso diferenciador entre un nosotros y ellos, deno- minado «otherness» (Hansen, 2006). En la necesidad de buscar elementos que cohesionen un grupo de seguridad a través de la identificación como lo es la UNASUR, se crea un elemento diferenciador con agentes que son excluidos del proyecto de comunidad de seguridad. Esta diferenciación se expresa a través de prácticas discursivas y acciones concretas que buscan alejar a ese otro. La necesidad de crear cierta identificación con el grupo lleva a que este genere un othering ya sea presentando al otro como una amenaza o como rival, o simplemente como un ente diferente que no presenta riesgos para el grupo o actor que constituye el nosotros (Wehner, 2010: 8-10).

En consecuencia, la UNASUR y su CDs son solo una parte de una agenda más amplia de gobernanza regional en temas de defensa y seguridad. Sin embargo, debido a que estos distintos niveles de gobernanza están entrelazados, se generan mecanismos sinérgicos respecto de cuáles experiencias bilaterales son importadas por el sistema multilateral-regional de gobernanza y a la inversa. Como la unasur y su CDS son solo una parte del sistema de gobernanza regional se hace necesario analizar desde una perspectiva más amplia los intereses nacionales, las diferencias ideológicas de sus miembros, los conflictos y las crisis internas y nacionales, el rol de los actores e instituciones externas que constituyen y dan forma a una agenda de seguridad en su sentido amplio, pero que igualmente afectan el nivel regional de seguridad. 
4. ACTORES Y CRISIS REGIONALES

\subsection{Las agendas nacionales frente a la UNASUR y el CDS}

Brasil es el autor intelectual de la UNASUR, así como el principal agente en dar vida al CDS. Detlef Nolte (2009: 15-16) analiza cómo los poderes regionales crean mecanismos de gobernanza regional ya que estos sirven como base para la proyección de poder del líder regional -hard y soft power. Por lo tanto, la creación de la UNASUR y el CDS es una forma de legitimar la aspiración brasileña de obtener un asiento permanente en el Consejo de Seguridad de las Naciones Unidas (Gratius, 2008: 3; Flemes, 2010: 102). En este sentido, la creación y el proceso de desarrollo de la UNASUR son una forma de delimitar la esfera de influencia de Brasil de modo de favorecer sus intereses regionales y globales (Medeiros Filho, 2009: 11-12). Sin embargo, los intereses brasileños por ejercer un rol de poder a nivel global así como un rol preponderante en UNASUR son vistos con cierta distancia por los llamados poderes secundarios o intermedios de la región (Flemes, 2010).

Un caso concreto es el de Venezuela y su líder Hugo Chávez, quien ha hecho de la UNASUR y su CDS un instrumento para sus intereses más que para el propio Brasil (Malamud, 2010: 75-76). En este sentido, Brasil ha creado la necesidad de la UNASUR y del CDS pero ha dejado a otros el rol articulador y de funcionamiento de la institución, lo que coincide con la visión de Brasil como poder hegemónico consensual (Burges,
2008). El interés de Venezuela ha sido apoyar la creación de una institución de cooperación en defensa y seguridad: incluso en 2003 propuso crear una OTANsudamericana. Por lo tanto, el apoyo venezolano en la creación y posterior desarrollo de competencias del cDs ha sido manifiesto. De hecho, la UNASur es, hasta cierto punto, una iniciativa dual de Venezuela y Brasil (Flemes, 2007). Sin embargo, sus motivaciones y agendas son distintas. Para el primero, la UNASUR y el CDS son una forma de realzar su misión y rol de promotor de valores anti-imperialistas en Sudamérica, mientras que para el segundo es una plataforma de inserción y de aceptación como poder global (Gratius, 2008: 5). Para Oscar Medeiros Filho (2009: 9) esta agenda venezolana respecto de UNASUR es la agenda bolivariana, que es apoyada por Bolivia y Ecuador.

Para Argentina, la UNASUR y el cDses una forma de mantener su presencia como poder equilibrador de Brasil y de seguir siendo un socio privilegiado en la agenda exterior de ese país. De hecho, Argentina ve con escepticismo la posibilidad de que Brasil pueda ejercer un liderazgo hegemónico en la UNASUR y el CDS para validar sus aspiraciones globales y apuesta en cambio por una agenda de seguridad Mercosuriana, consistente en promover principios de estabilidad democrática y la solución rápida de conflictos que pongan en riesgo gobiernos democráticamente elegidos. Al respecto, la idea es extrapolar la experiencia de cooperación de mercosur a la unASUR (Madeiros 
Daniel Flemes, Detlef Nolte y Leslie Wehner • Una comunidad de seguridad regional...

Filho, 2009: 10). Sin embargo, a la Argentina le interesa en especial que la percepción de un principio de igualdad o de compartir con Brasil el liderazgo en MERCOSUR pueda también plasmarse en ser la UNASUR. Un ejemplo de esta visión de liderazgo compartido es la elección de Néstor Kirchner como Secretario General de la unASur con el apoyo de Brasil (La Nación, 2010).

A pesar de que Oscar Medeiros Filho (2009) menciona tres tipos de agenda de seguridad en UNASur y su CDS, ${ }^{1}$ puede agregarse un cuarto, compartido por Colombia, Perú y Chile. Este cuarto tipo de agenda puede denominarse una agenda de seguridad y de autonomía abierta, cuyo objetivo es complementar y mantener los canales de diálogo y cooperación con todos los países del continente americano, especialmente con Estados Unidos y México. Si bien es cierto que la Presidenta Michelle Bachelet (2006-2010) ejerció la presidencia pro-tempore de UNASUR (mayo de 2008 a agosto de 2009), Chile ve con cierta distancia los proyectos de integración regional en general y prioriza como eje complementario la integración bilateral (ver Fernández, 2010: 4). Dentro de los aspectos salientes de la política exterior del gobierno chileno se cuenta el fortalecimiento del rol de la OEA, más allá del hecho de que su Secretario General sea José Miguel Insulza (2005 a la fecha). Además, Chile busca tener una relación pragmática con los Estados Unidos en todos los ámbitos diplomáticos, in-

1 Brasileña, Bolivariana y Mercosuriana. cluyendo aquellos del área de defensa y seguridad. En este sentido, Estados Unidos ve a Chile como aliado en la región sudamericana, además de como uno de sus mayores compradores de equipo bélico en la región.

Chile ve en el liderazgo brasileño una forma de contener a Hugo Chávez, dadas las diferencias ideológicas con el presidente de Chile, Sebastián Piñera (2010 a la fecha). Al adoptar una postura aperturista o de complementación de la UNASUR a otros foros regionales como la oEA, Chile persigue no dejar fuera a México, que con el tiempo podría servir para balancear a Brasil en caso de que este pretenda posiciones de excesiva supremacía (La Tercera, 2010a: R14). Por otra parte, el hecho de que en la UNASUR haya fuerzas ideológicas centrífugas hace que a Chile se le asocie con el grupo de países neoliberales -Colombia y Perú. Pese a estas diferencias, Chile ha buscado jugar un rol de líder emprendedor e intelectual junto a Argentina en el CDS, ya que ambos han llevado a este Consejo sus experiencias exitosas de cooperación bilateral en seguridad y defensa (sobre liderazgo: Young, 1991). Además, Chile aprobó la idea de acordar un sistema de homologación de gastos militares y de eliminación de hipótesis de conflicto para mejorar su relación con Perú. Esto se enmarcó en la institucionalidad del CDS y las medidas de confianza mutua que este Consejo busca promover. ${ }^{2}$ Sin embargo, al parece ser esta es una

2 Ver sección 2. 
forma de institucionalizar sus intereses en el ámbito regional y de mejorar una complicada relación bilateral con Perú.

Colombia es un actor relevante que tiene una visión escéptica sobre el rol de la UNASUR y del CDS como ente exclusivo de gobernanza de seguridad. Como ya se señaló, este país fue bastante reacio a formar parte del CDS debido a la alianza estratégica de Colombia con Estados Unidos en la lucha contra el narcotráfico (Plan Colombia, reforzado por la alianza para derrotar a grupos terroristas transnacionales (Carvajal y Pardo, 2002: 200-219; Tokatlian, 2009: 229). De hecho, por motivos de seguridad y de distancia ideológica con Ecuador y Venezuela, Colombia busca prevenir un entrampamiento exclusivo en la UNASUR, donde puede verse disminuido frente a amenazas y conflictos con Venezuela y Ecuador. ${ }^{3}$

Lo que genera mayor recelo en el resto de los países es que Colombia haya autorizado a Estados Unidos la instalación de siete bases militares en su territorio para cooperar en la lucha contra el narcotráfico. Estas bases no solo producen vulnerabilidad en los países fronterizos, incluido Brasil y la sensación de que Estados Unidos quiere influir directamente en asuntos de seguridad en la región sino que generan problemas fronterizos como el de la incursión colombiana en territorio ecuatoriano para desarticular a un

Pese a esta reticencia hacia la UNASUR y su CDS, Colombia presentaría un candidato/a para el cargo vacante de Secretario General de la UnASUR (El Tiempo 2011). grupo de las FARC en 2008. Ecuador y Venezuela tienen la impresión de que este acto de violación de soberanía fue articulado por Colombia en conjunto con los servicios de inteligencia estadounidenses, impresión que genera un clima mayor de conflicto y de divergencia ideológica dentro del CDS (ver Flemes y Radseck 2009: 14). En otras palabras las hipótesis de conflicto siguen estando presentes en la región y parten desde la relación Colombia-Venezuela-Ecuador.

\subsection{El rol mediador de la UNASUR y su CDS}

Entre 2008 y 2010 se han producido cuatro crisis internas e interestatales en Sudamérica en las cuales la UNASUR ha intervenido para buscar una solución pronta y pacífica:

\section{Crisis de Bolivia}

Este país vivió una crisis institucional en septiembre de 2008. Las Provincias de Santa Cruz, Beni, Pando y Tarija declararon su autonomía del gobierno central del Presidente Evo Morales. El conflicto interno causó a lo menos 30 muertes en enfrentamientos entre los simpatizantes del gobierno y los que apoyaban la autonomía. La UNASUR a través del liderazgo de Michelle Bachelet, que ejercía la presidencia pro-tempore, convocó al organismo para buscar una solución pacífica al conflicto. De hecho, el episodio constituyó el debut de la UNASUR como ente proveedor de 
Daniel Flemes, Detlef Nolte y Leslie Wehner • Una comunidad de seguridad regional...

seguridad y promotor de la paz regional (Flemes y Radseck, 2009: 25).

La declaración de La Moneda -septiembre de 2008- no solo resalta que el grupo regional no toleraría intentos por disgregar la unidad territorial y soberana del Estado de Bolivia sino que manifiesta una exclusión discursiva de otros actores posibles como la OEA y Estados Unidos. Asimismo, expresa el compromiso de los países por respaldar al gobierno democráticamente elegido en el país y pone de relieve principios rectores del tratado constitutivo de la UNASUR, como el respeto irrestricto de la soberanía, la no intervención en los asuntos internos de los Estados miembros y la integridad e inviolabilidad territorial (ver Declaración Presidentes UNASUR, 2008).

Los países líderes de la UNASUR se valieron del éxito en el manejo de esta crisis para asentar un discurso de legitimidad y de necesidad de contar con un órgano como este cuyo rol sea servir de mediador en conflictos regionales. Además, los países de la UNASUR articularon una retórica de cómo el grupo regional puede encargarse de proveer un bien como la preservación de la democracia y la paz en la región. En el discurso oficial se destaca expresamente la madurez de los países sudamericanos para hacer frente a sus propios problemas sin la ayuda de terceros (Malamud, 2008). Este discurso de responsabilidad y legitimidad de la UNASUR incluso fue presentado en foros multilaterales como la Asamblea General de las Naciones Unidas en 2008 por los presidentes de Argentina, Brasil, y Chile y por el embajador de Venezuela ante la Organización (ver Fernández, 2008; Lula da Silva 2008; Bachelet, 2008; Chaderton Matos, 2008).

\section{El problema de las bases estadounidenses en Colombia}

El acuerdo entre Colombia y Estados Unidos en virtud del cual se permite que las tropas estadounidenses utilicen bases colombianas generó rechazo en los países de la región, especialmente los que limitan con Colombia. La autorización para que Estados Unidos utilice las bases militares perseguía profundizar la alianza en la lucha contra el narcotráfico y el terrorismo transnacional, pero además respondió a una lógica económica. Está lógica se basa en que el Acuerdo Complementario para la Cooperación y Asistencia Técnica en Defensa y Seguridad facilitaría la aprobación del Tratado de Libre Comercio suscrito entre ambos países, pero que aún no ha sido aprobado por el Congreso americano (Flemes y Nolte, 2010: 10).

Para tratar el tema de las bases americanas en suelo colombiano, los países de la UNASUR convocaron a una reunión extraordinaria de jefes de Estado que se llevó a cabo en Bariloche, Argentina, en agosto de 2009. Desde el punto de vista de la institucionalización de la UNASUR y su CDS; los resultados de la reunión no fueron totalmente satisfactorios, pero la disposición de Colombia de participar en el foro y consensuar una 
declaración conciliadora junto a países como Venezuela puede considerars un logro, aunque limitado, de la UNASUR. Pese a las críticas por el pobre resultado de Bariloche, el presidente Lula señaló: «Yo salí de Bariloche convencido de que dimos otro paso. Después de muchas divergencias, llegamos a la conclusión de que podemos seguir peleando y discrepando, pero que tenemos que construir posiciones unitarias en torno de los asuntos que son importantes y delicados para nosotros» (Lula da Silva, 2009). El presidente de Ecuador, por su parte, catalogó la reunión como un éxito: «La reunión fue un éxito rotundo, fue dura porque el tema era álgido, pero acabó como mucha armonía, se aprobó una resolución por consenso, unánimemente, y creo que se discutieron los temas con energía pero con altura» (Correa, 2009). La racionalidad de estas declaraciones radica en la necesidad de sostener la legitimidad y profundizar la institucionalización de la UNASUR para resolver y mediar en crisis entre sus Estados miembros.

La declaración de Bariloche subraya la vocación de la UNASUR por «la solución pacífica de las controversias y la promoción del diálogo y el consenso en materia de defensa...» También reafirma los compromisos de «...fortalecer la lucha contra el terrorismo y la delincuencia transnacional organizada y sus delitos conexos: el narcotráfico...» Por último, busca «reafirmar que la presencia de fuerzas militares extranjeras no puede, con sus medios y recursos vinculados a objetivos propios, amenazar la soberanía e integridad de cualquier nación suramericana y en consecuencia la paz y seguridad en la región» (ver unAsur, 2009).

A pesar del tono conciliador de la declaración de Bariloche, en una reunión posterior de Ministros de Defensa y de Relaciones Exteriores celebrada en Quito en septiembre de 2009, Colombia se negó a dar detalles de su acuerdo con Estados Unidos e incluso amenazó con retirarse de la UNASUR al verse arrinconado por las peticiones de sus pares (Flemes y Nolte, 2010: 11). Sin embargo, la declaración de los Ministros nuevamente subraya el rol de la UNASUR como foro de discusión y resolución de conflictos y el hecho de que el bloque es un instrumento para promover y alcanzar la paz regional (ver Consejo de Defensa Suramericano, 2009b).

\section{La crisis entre Colombia y Venezuela}

El conflicto entre estos dos países estalló en julio de 2010, aunque el origen de las malas relaciones entre los dos países data de 2008 durante el conflicto Ecuador-Colombia, en que Venezuela apoyó a Ecuador. El origen inmediato de la crisis fue la solicitud del presidente de Colombia Álvaro Uribe a sus vecinos de que prohibiera el ingreso de las FARC a sus respectivos territorios. Además, el gobierno de Colombia anunció la posibilidad de acudir a instancias multilaterales para denunciar a Venezuela por la presunta presencia de las FARC 
Daniel Flemes, Detlef Nolte y Leslie Wehner • Una comunidad de seguridad regional...

en su territorio. De hecho, Colombia formalizó su denuncia contra Caracas ante la OEA, pidiendo que se estableciera una comisión internacional para verificar el hecho. De acuerdo con la denuncia, habría hasta 1500 miembros de la FARC en territorio venezolano (Libertaddigital, 2010). Esto significaba que la oeA debía nombrar una comisión para inspeccionar el hecho en territorio venezolano. Ante esta denuncia de Colombia en la oeA, Hugo Chávez rompió relaciones diplomáticas con el gobierno de su vecino y acusó a Estados Unidos de querer atacar a Venezuela desde Colombia (El Mundo, 2010). Al incluir a Estados Unidos en el problema y desvirtuar el rol de la OEA en la comprobación del mérito de las denuncias de Colombia, la UNASUR y su CDS pasaron a ser el único ente legítimo -desde la perspectiva venezolana- para intervenir y buscar salida al conflicto (Latin American Weekly Report, 2010: 4-5). Sin embargo, esto se tradujo en una presión adicional sobre Colombia para que la UNASUR fuese la encargada de coordinar y facilitar el diálogo bilateral a fin de prevenir escaladas que lleven a un eventual uso de la fuerza.

Brasil y Argentina vieron esta deslegitimación de la oEA como una oportunidad para seguir institucionalizando el rol mediador de la UNASUR y su CDS. En rigor, Brasil asumió un liderazgo menos mediático, dejando las cámaras para un debut como líder promotor del diálogo al recién elegido Secretario General de la UNASUR, Néstor Kirchner. De hecho, fue este quien apareció junto al recién asumido Presidente de Colombia Juan Manuel Santos y a Hugo Chávez en la reunión de Santa Marta el 10 de agosto de 2010, donde ambos países reanudaron sus relaciones diplomáticas, buscando recomponer la confianza mutua (El Clarín, 2010). ${ }^{4}$

Las imágenes de la presencia del Secretario General de la UNASur y el significado discursivo que a estas se le atribuyen ayudan a definir los roles y espacios de la nueva influencia de este grupo en relación con sus pares, la oea y los Estados Unidos. Aquí se entrelazan dos fenómenos como son la necesidad de reducir el papel de la oeA y de Estados Unidos en la región-balance institucional y de poder, y la necesidad de excluir y remarcar diferencias entre 'nosotros' y 'ellos' en un sentido discursivo que impacta no solo en la posible formación de una identidad conjunta en materias de seguridad sino que ayuda a legitimar y profundizar la necesidad de seguir institucionalizando las prácticas de la UNASUR y el CDS. Al respecto, el canciller argentino Héctor Timerman señala, excluyendo al otro: "Es importante que sea la región la que resuelva los conflictos»; mientras que el líder

4 Además, el 29 de julio de 2010 tuvo lugar en Quito una reunión extraordinaria de ministros de relaciones exteriores de la UNASUR que no produjo resultados concretos. Sin embargo, la retórica desde Ecuador -presidencia pro tempore- una vez que se solucionó el conflicto en la reunión de Santa Marta fue recalcar que en la reunión de Quito se habían sentado las bases para el diálogo y posterior solución del conflicto (La Tercera, 2010b: 13); es decir, se muestra a la UNASUR como un proceso. 
brasileño destaca el principio de que se trata de un nosotros todos (UNASUR) y no solo 'uno' el que asume responsabilidades: «Brasil reitera su disposición a cooperar en el ámbito de UNASUR", y expresó su satisfacción por el rol del Secretario General para resolver el conflicto (La Tercera, 2010b: 13). En tanto, el presidente Juan Manuel Santos destacó la labor del Secretario General de UNASUR como seria y silenciosa (en La Tercera, 2010c: 14). Por último, el Presidente de Venezuela Hugo Chávez destacó y validó el rol de la UNASUR personificado en la figura de su Secretario General Néstor Kirchner, «No es poca cosa» que el nuevo organismo regional... haya logrado conjurar la posibilidad cierta de una guerra fraticida... [Néstor Kirchner] merece toda nuestra gratitud y nuestro reconocimiento por su paciente, tesonero y eficaz ejercicio de mediación» (en El Universal, 2010).

\section{El intento de golpe en Ecuador}

El 30 de septiembre de 2010 la policía nacional ecuatoriana salió a las calles a protestar para presionar al Presidente Rafael Correa a que vetara la ley de servicio público que afectaría los derechos del cuerpo policíaco (Brombacher y Maihold 2010: 7). Al tratar de mediar directamente y luego de que explotara una bomba lacrimógena cerca de su rostro, el Presidente Rafael Correa fue llevado a un Hospital de la Policía en Quito, donde estuvo retenido. De hecho, el Presidente Correa tuvo que ser rescatado mediante una operación coordinada entre militares y un grupo de elite de la policía.

Este episodio llevó a la UNASUR a jugar nuevamente un rol de resolución de conflictos. De hecho, los presidentes sudamericanos se reunieron de emergencia en Buenos Aires el mismo día de los hechos y emitieron una declaración condenando el intento de golpe. En la declaración se hace explícito el apoyo a la democracia y se anuncian medidas de presión como el cierre de fronteras y la aplicación de sanciones comerciales para reestablecer el orden democrático en el país. Además, se decide acelerar la preparación de una cláusula democrática en un Protocolo adicional al Tratado constitutivo de la UNASUR. Los distintos países decidieron asimismo enviar a sus ministros de relaciones exteriores a Quito el mismo 1 de octubre de 2010, día de la Declaración de Buenos Aires, como forma de manifestar el total respaldo al Presidente constitucional (ver UNASUR 2010).

Pese a que por conducto de su Consejo Permanente la oEA repudió y condenó el fallido intento y países como Brasil manifestaron que tanto la UNASUr, como el mercosur y la oea debían dar una respuesta coordinada frente al hecho, lo cierto es que el rol de la UNASUR fue más notorio porque los presidentes sudamericanos se reunieron en el marco de la UNASUR para resolver este conflicto -balance institucional y othering. Además, es importante señalar que en la declaración de Buenos Aires se adoptan prácticas concretas para el caso de que se produzcan crisis 
Daniel Flemes, Detlef Nolte y Leslie Wehner • Una comunidad de seguridad regional...

en el futuro y la UNASUR como tal adoptó formalmente un rol de defensor de la democracia y de plataforma para la resolución conflictos al enunciar medidas de presión y de no tolerancia frente a nuevos intentos golpistas. Si bien esta declaración se relaciona con el caso de Ecuador, sus principios y medidas son universales y aplicables a casos similares (ver punto 4, UNASUR 2010).

\section{ConClusión}

La UNASUR y su CDS responden a la lógica de buscar proveer seguridad mediante la coordinación creciente de las agendas nacionales de seguridad y defensa. En el sentido propuesto por Adler y Barnett (1998), la intencionalidad de los países líderes de este organismo es sentar las bases para el desarrollo de una comunidad de seguridad de tipo «rigurosa». En el discurso de los países líderes de la UNASUR, así como en el tratado constitutivo de este grupo regional y en el plan de acción del CDs 2010-2011, lo que se busca es crear mecanismos de coordinación que sedimenten y creen rutinas institucionalizadas en el manejo de temas comunes de seguridad y defensa.

A pesar de que en Sudamérica siguen presentándose hipótesis de conflicto entre Estados, como lo demuestra la crisis entre Venezuela y Colombia, se podría afirmar que la UNASUR y el CDS han dejado atrás la forma naciente para adoptar los patrones de una comunidad de seguridad en su sentido riguroso e iniciar una etapa ascendente. Paradójicamente, han sido las crisis en las cuales la UNASUR ha mediado, las que han generado en gran parte este proceso de transición de naciente a ascendente. Se dan tres elementos que se complementan: primero, la necesidad de equilibrar y excluir institucionalmente a la OEA y a los Estados Unidos por mucho que los miembros de la UNASUR también sean parte de este otro órgano internacional; segundo; se generan discursos y retóricas de legitimidad y necesidad de profundizar las prácticas de la UNASUR y su CDS para mediar en crisis futuras; y tercero, se generan discursos diferenciadores entre un «nosotros» (países de UNASUR) y «los demás» (no-miembros), aunque aún se está lejos de hablar de una identidad sudamericana de defensa y seguridad.

También existen hechos menos mediáticos de coordinación y de búsqueda de prácticas de institucionalización en temas de seguridad y defensa que han contribuido fuertemente a la transición naciente-ascendente. Entre ellos cabe mencionar la aprobación de mecanismos y políticas de creación de confianzas mutuas, que han materializado en políticas concretas como la adopción de sistemas de homologación de gastos militares entre Perú y Chile en el marco del CDsy la planificación de ejercicios militares conjuntos ante hipótesis de misiones humanitarias y de mantenimiento de la paz.

Los países líderes de la UNASUR tienen intereses y agendas divergentes que hasta el momento no han sido un 
impedimento mayor para avanzar en una agenda de seguridad debido a la lógica de balances de poderes internos. De hecho, para el líder regional-Brasilla UNASUR es una forma de legitimar sus aspiraciones globales, mientras que para países intermedios representa una forma de limitar una posible supremacía desmedida de Brasil. Por lo tanto, aunque la UNASUR y el CDS distan mucho de ser una comunidad de seguridad madura, a través de su manejo de crisis, del uso discursivo de estas y de la necesidad material de sus países líderes de excluir agentes y agencias externas están avanzando e institucionalizando en conjunto prácticas y rutinas formales e informales que constituyen una agenda de seguridad y defensa regional.

\section{BIBLIOGRAFÍA}

Adler, Emmanuel y Michael Barnett (1998), "A framework for the study of security communities», en Adler, Emanuel y Michael Barnett (eds.) Security Communities, Cambridge, Cambridge University Press, pp. 29-65.

BACHelet, Michelle (2008), Intervención ante la $63^{\circ}$ Asamblea General de las Naciones Unidas, en Nueva York; 24 de septiembre, (http://www.lanacion.cl/ prontus_noticias_v2/site/artic/20080924/ pags/20080924124048.html), consultado el 26 agosto de 2010.

Bellamy, Alex (2004), Security Communities and their Neighbours: Regional Fortresses or Global Integrators?, Basingstoke, Palgrave Macmillan.

BROMBACHER, Daniel y Gunther Maihold (2010), Die Grenzen charismatischer politischer Führung Lateinamerikas Linke vor neuen Kursbestimmungen, swp-Aktuell 2010/A 71.
Burges, Sean (2008), "Consensual Hegemony: Theorizing the Practice of Brazilian Foreign Policy after the Cold War», International Relations 22(1): 65-84.

Carvajal, Leonardo y Rodrigo Pardo (2002) «La Internacionalización del Conflicto Doméstico y los Procesos de Paz (Historia Reciente y Principales Desafíos)», en Ardila, Martha; Diego Cardona, Arlene Tickner (eds.) Prioridades y Desafíos de la Política Exterior Colombiana, Bogotá, Friedrich Ebert-Stiftung y Hanns Seidel Stiftung, pp. 181-236.

Chaderton Matos, Roy (2008), Intervención del Emabajador Extraordinario y Plenipotenciario Roy Chaderton Matos, enviado especial del Presidente de la República Bolivariana de Venezuela para el $63^{\circ}$ período ordinario de sesiones de la Asamblea General de las Naciones Unidas; 29 de septiembre; en (http://www.un.org/ ga/63/generaldebate/pdf/venezuela_es.pdf) consultado el 27 de agosto de 2010.

Consejo de Defensa Suramericano (2010a), Suramérica avanza en la construcción de un modelo para medir gastos de defensa; Noticias 03 agosto, (www.cdsunasur.org) consultado el 22 de agosto de 2010.

Consejo de Defensa Suramericano (2010c), Plan de Acción 2010-11. (http:// www.cdsunasur.org/en/action-plan/plande-accion-2010-2011) consultado el 27 de agosto de 2010.

Consejo de Defensa Suramericano $\left(2009^{\mathrm{a}}\right)$, UNASUR approves mutual confidence-building unprecedented mechanism. Noticias 28 de noviembre, (http://www. cdsunasur.org/en/news/178-reunionministros) consultado el 20 de agosto de 2010.

Consejo de Defensa Suramericano (2009b) Reunión Extraordinaria de Ministros de Relaciones Exteriores y Defensa de la UNASUR, septiembre y noviembre 2009, (www.cdsunasur.org/en/consejo-de-defensa-suramericano/official-documents/57espanol/150-reunion-extraordinaria-deministros-de-relaciones-exteriores-y-de- 
Daniel Flemes, Detlef Nolte y Leslie Wehner • Una comunidad de seguridad regional...

defensa-de-la-unasur) consultado el $23 \mathrm{de}$ agosto de 2010).

Correa, Rafael (2009), «Rafael Correa califica de «exitosa» cumbre de Unasur», en El Nuevo Diario, 29 agosto, (http:// www.elnuevodiario.com.ni/internacionales/55862) consultado el 26 de agosto de 2010.

Declaración de Presidentes UNASUR por crisis en Bolivia (2008), 15 de septiembre, (http://lta.reuters.com/article/domesticNews/idLTAN1533709020080916) consultado el 26 de agosto de 2010.

Deutsch, Karl (1957), Political Community and the North Atlantic Area, Princeton, Princeton University Press.

El Clarín (2010), Destacan el papel de Kirchner y Lula como mediadores, 10 agosto, (http://www.clarin.com/mundo/ america_latina/Destacan-papel-KirchnerLula-mediadores_0_314368636.html) consultado el 23 de agosto de 2010.

El Mundo (2010), Colombia y Venezuela, una historia de amor y odio, 10 agosto 2010, (http://www.elmundo.es/ameri$\mathrm{ca} / 2010 / 08 / 11 /$ venezuela/1281494971. html) consultado el 26 de agosto de 2010.

El Tiempo (2011), Colombia y Venezuela 'pujarán’ por la secretaría general de UNASUR, 20 de enero, (http://www.eltiempo.com) consultado el 20 de enero de 2011.

El Universal (2010), Chávez destaca papel de Unasur en normalización con Colombia, 15 agosto, (www.eluniversal. com/2010/08/15) consultado el 27 de agosto de 2010.

Fernández, Cristina (2008), Palabras de la Presidenta de la Nación, Cristina Fernández de Kirchner en la apertura de la Asamblea General de las Naciones Unidas, en la ciudad de Nueva York, 23 septiembre, (http://www.un.org/ga/63/generaldebate/ pdf/argentina_es.pdf) consultado el $26 \mathrm{de}$ agosto de 2010.

Fernández, Mariano (2010), "Chile y el desafío de UNASURR», La Tercera, 7 Julio p. 4, (www.latercera.com) consultado el 7 de julio de 2010.
Flemes, Daniel (2010), «Brazil: Strategic Options in the Changing World Order», en Flemes, Daniel (ed.), Regional Leadership in the Global System: Ideas, Interests and Strategies of Regional Powers, Aldershot, Ashgate, pp. 93-112.

Flemes, Daniel y Detlef Nolte (2010), «Alianzas externas para armamento y defensa: Una nueva dimensión en la agenda de seguridad latinoamericana", Foreign Affairs Latinoamérica 10(1), pp. 1-13.

Flemes, Daniel y Thorsten Wojczewski (2010), "Contested Leadership in International Relations: Power Politics in South America, South Asia, and Sub-Saharan Africa», GIGA Working Papers 121, Hamburgo, German Institute of Global and Area Studies.

Flemes, Daniel y Detlef Nolte (2009), «Externe Rüstungs- und Militärallianzen: Eine neue Dimension in Lateinamerikas Sicherheitsagenda ", GIGA Fokus -Lateinamerika, no. 9, Hamburgo, German Institute of Global and Area Studies.

Flemes, Daniel (2007), «Brasilien - Regionalmacht mit globalen Ambitionen ", GIGA Focus Lateinamerika no. 6, Hamburgo, German Institute of Global and Area Studies.

Flemes, Daniel y Michael Radseck (2009), "Creating Multilevel Security Governance in South America», GIGA Working Papers 117, Hamburgo: German Institute of Global and Area Studies.

Gratius, Susanne (2008), ¿Hacia una OtAN sudamericana? Brasil y un Consejo de Defensa Sudamericano. Comentario-Abril, Madrid, FRIDE.

Hansen, Lene (2006), Security as Practice: Discourse Analysis and the Bosnian War, Oxon, Routledge.

He, Kai (2008), «Institutional balancing and international relations theory: economic interdependence and balance of power strategies in Southeast Asia», European Journal of International Relations 14, no 3, pp. 489-518.

Hurrell, Andrew (2005), «Hegemony and Regional Governance in the Americas», 
en Fawcett, Louise y Mónica Serrano (eds.), Regionalism y Governance in the Americas. Continental Drift, Basingstoke, Palgrave, pp. 185-208.

Keohane, Robert (1989), «Neoliberal Institutionalism: A perspective on world politics», en Keohane, Robert, International Institutions and State Power: Essays in International Relations Theory, Boulder, Westview Press, pp. 1-20.

La Nación (2010), Kirchner juró como secretario general de la Unasur y Mujica admitió «el costo político» de acompañar la designación, 4 Mayo. (http://www.lanacion.com.ar/nota.asp?nota_id=1261034) consultado el 14 de agosto de 2010.

La Tercera (2010a), La agenda vecinal. 8 de agosto, p. R14, (www.tercera.cl) consultado el 8 de agosto de 2010.

La Tercera (2010b), Rol de Unasur en crisis entre Colombia y Venezuela resta terreno a la OEA. 12 Augusto, p 13, (www.latercera. cl) consultado el 12 de agosto de 2010.

La Tercera (2010c), Chávez y Santos restablecen relaciones y acuerdan plan para combatir en conjunto a las Farc, 11 agosto, p. 14, (www.latercera.cl) consultado el 11 de agosto de 2010).

Latin American Weekly Report (2010), Chávez skilfully alters nature of debate. 29 julio, WR-10-30; pp. 4-5.

Libertaddigital (2010), Colombia revela que hay hasta 1.500 terroristas de las FARC en Venezuela, 22 julio, (http://www.libertaddigital.com/mundo/colombia-revelaante-la-oea-que-hay-1500-terroristasde-las-farc-en-venezuela-1276397977/) consultado el 26 de agosto de 2010.

Lula DA Silva, Luiz Inácio (2009), Lula dice que en Unasur se dio paso importante para la integración, en elespectador.com, 31 agosto (http://www.elespectador. com/noticias/elmundo/articulo158836lula-dice-unasur-se-dio-paso-importanteintegracion) consultado el 26 de agosto de 2010.

Lula DA Silva, Luiz Inácio (2008), Statement by H.E. Luiz Inácio Lula da Silva, President of the Federative Republic of Brazil, at the General Debate of the $63^{\text {rd }}$ Session of the United Nations General Assembly, (http://www.un.int/brazil/speech/008dCS-agnu-Open \%20debate \% 20230908. html) consultado el 26 de agosto de 2010. Malamud, Andrés (2010), « ¿Mejor solo que mal acompañado? Brasil, entre la turbulencia regional y la emergencia global», en Anuario Iberoamericano, Madrid, Ediciones Pirámide, pp. 71

Malamud, Carlos (2008), La Cumbre de Unasur en Santiago de Chile y el conflicto en Bolivia, ARI-no 121/2008, Madrid, Real Instituto Elcano.

Medeiros Filho, Oscar (2009), Conselho de Defensa Sudamericano: Origens, Demandas e Propósitos. Trabajo presentado en el III Encontro Nacional da Associação Brasileira de Estudos de Defensá-ABED, 28-31 julio, Londrina.

NABERs, Dirk (2010), «Power, Leadership and Hegemony in International Politics», en Flemes, Daniel (ed.) Regional Leadership in the Global System: Ideas, Interests and Strategies of Regional Powers, Aldershot, Ashgate, pp. 92-124.

Nolte, Detlef (2009), Regional Power and Regional Governance. Trabajo presentado en la Conferencia del Regional Powers Network, Paris 8-9 octubre.

Presidencia Pro-Tempore Ecuador (2010), Informe de Gestión 2009-2010. Consejo de Defensa Suramericano -UNASUR.

Shifter, Michael y Adam Siegel (2010), «Colombia y Ecuador en 2009: el tortuoso camino hacia un restablecimiento de las relaciones", en Anuario Iberoamericano, Madrid: Ediciones Pirámide, pp. 87-102.

Statute of the Unasur South American Defense Council (2008), 11 de diciembre, (http://www.cdsunasur.org/en/ consejo-de-defensa-suramericano/officialdocuments/57-espanol/159-estatutodel-consejo-de-defensa-suramericano) consultado el 22 de agosto de 2010.

TAvares, Rodrigo (2010), Regional Security: The capacity of international organizations, Abingdon, Routledge. 
Daniel Flemes, Detlef Nolte y Leslie Wehner • Una comunidad de seguridad regional...

Tokatlian, Juan Gabriel (2009), La construcción de un «Estado fallido» en la política mundial: el caso de las relaciones entre Estados Unidos y Colombia, en Hirst, Monica (ed.) Crisis del Estado e Intervención Internacional, Buenos Aires, Edhasa, pp. 207-248.

Tratado Constitutivo De la unasur (2008), 16 diciembre, (http://www.cdsunasur.org/en/consejo-de-defensa-suramericano/official-documents/57-espanol/160tratado-constitutivo-de-la-unasur) consultado el 21 de agosto de 2010.

Ugarte, José Manuel (2010), «El Consejo de Defensa Suramericano: Naturaleza, Balance Provisorio, Perspectivas y Desafíos», en Mathieu, Hans y Catalina Niño Guarnizo (eds.) Anuario 2010 de la Seguridad Regional en América Latina y el Caribe, Bogota, Friedrich Ebert Stiftung, pp. 25-42.

unASur (2010), Declaración de Buenos Aires sobre la situación en Ecuador, 1 octubre, (http://www.pptunasur.com/contenidos. php? id=1089\&tipo=5\&idiom $=1$ ) consultado el 20 de enero de 2011.

UNASUR (2009), Reunión Extraordinaria de Jefas y Jefes de Estado y de Gobierno de la UNASUR, 28 agosto, (http://www.cdsunasur. org/en/consejo-de-defensa-suramericano/ official-documents/57-espanol/340-reunion-extraordinaria-de-jefas-y-jefesde-estado-y-de-gobierno-de-la-unasur) consultado el 24 de agosto de 2010.

WeHNER, Leslie (2010), «From rivalry to mutual trust: the othering process between Bolivia and Chile», GIGA Working Papers 135, Hamburgo, German Institute of Global and Area Studies.

Young, Oran (1991), «Political Leadership and Regime Formation: On the Development of Institutions in International Society", International Organization 45, pp. 281-308. 